\title{
Rényi-Fisher entropy product as a marker of topological phase transitions
}

\author{
J. C. Bolívar \\ Departamento de Física Atómica, Molecular y Nuclear and Instituto Carlos I de \\ Física Teórica y Computacional, Universidad de Granada, Fuentenueva s/n 18071 \\ Granada, Spain \\ E-mail: jcbolivar@ugr.es
}

\begin{abstract}
Ágnes Nagy
Department of Theoretical Physics, University of Debrecen, H-4002 Debrecen, Hungary

E-mail: anagy@phys . unideb.hu
\end{abstract}

\section{Elvira Romera}

Departamento de Física Atómica, Molecular y Nuclear and Instituto Carlos I de Física Teórica y Computacional, Universidad de Granada, Fuentenueva s/n 18071

Granada, Spain

E-mail: eromera@ugr.es

16 November 2017

\begin{abstract}
The combined Rényi-Fisher entropy product of electrons plus holes displays a minimum at the charge neutrality points. The Stam-Rényi difference and the Stam- Rényi uncertainty product of the electrons plus holes, show maxima at the charge neutrality points. Topological quantum numbers capable of detecting the topological insulator and the band insulator phases, are defined. Upper and lower bounds for the position and momentum space Rényi-Fisher entropy products are derived.

PACS numbers: $03.65 . V f, 03.65 . P m$,
\end{abstract}

\section{Introduction}

Information theoretical concepts have significant impact on research in physics. In this paper we show how Rényi-Fisher entropy product [1] can recognize topological phase transitions. Two-dimensional topological insulators have attracted attention in last years. They were theoretically predicted by Kane and Mele [2] using a two dimensional material with a spin-orbit interaction and was observed experimentally in several materials (see $[3,4]$ and references therein). The 2D Dirac equation has been used to describe low energy electronic properties of several topological 
insulators; among others 2D gapped Dirac materials such as silicene, germanene or tinene. These 2D materials were studied both experimentally and theoretically $[5,6,7,8,9,10,11,12,13,2,14,15,16,17,18,19,20,21]$ and they are similar to graphene but with a bluckled structure (with buckling length $l$ and a significant spinorbit coupling, $\Delta_{s o}$ ). This buckling length gives the possibility to control the structure by applying an electric field perpendicular to the layer and allows these systems undergo a topological phase transition (TPT) from a topological insulator (TI) to a trivial band insulator (BI). TI- BI transitions are characterized by a band inversion with a level crossing at some critical value of a control parameter (p. e. the electric field) .

It has already been shown that information theoretical concepts are powerful in analyzing quantum phase transitions [22, 23, 24, 25, 26, 27, 28]. Furthermore, it has also turned out that Shannon, Kullback-Leibler, Fisher information and Rényi entropies, uncertainty relations are distinctive indicators of topological phase transitions $[29,30,31,32,33,34,35]$. In this paper we point out that the Rényi-Fisher entropy product detects topological phase transitions. Namely, the combined Rényi-Fisher entropy product of electrons plus holes displays minimum at the charge neutrality points (CPA). Furthermore, Stam uncertainty relations and Stam- Rényi uncertainty products can be utilized in constucting quantities for electrons and holes that exhibit maxima at CPA. Upper and lower bounds for the position and momentum space Rényi-Fisher entropy products are derived. Moreover, we define topological quantum numbers capable of detecting the topological insulator and the band insulator phases.

The layout of the paper is as follows: topological phase transitions in 2D gapped Dirac materials is reviewed in the following section. In Section 3, first, analytical results for the Rényi-Fisher entropy product are summarized, then calculations for the silicene are presented and a topological quantum number is defined. Section 4. presents Stam uncertainty relations and Rényi-Stam uncertainty products and topological quantum numbers. Conclusions can be found in the last section.

\section{Eigenvalue problem in the vicinity of the Dirac point}

Our investigation is limited to the vicinity of the Dirac point, where the effective Hamiltonian takes the form [13]

$$
H_{\xi s}=v_{F}\left(\sigma_{x} p_{x}-\xi \sigma_{y} p_{y}\right)-\frac{1}{2} \xi_{s} \Delta_{\text {so }} \sigma_{z}+\frac{1}{2} \Delta_{z} \sigma_{z} .
$$

The inequivalent corners $K$ and $K^{\prime}$ of the Brillouin zone are distinguihed by the parameter $\xi: \xi=1(\xi=-1)$ stands for $K\left(K^{\prime}\right) . \sigma_{j}$ denotes the Pauli matrices and $v_{F}$ is the Fermi velocity of the Dirac fermions. There is an external magnetic field $B$ and an electric field $\mathcal{E}_{z}$ applied perpendicular to the silicene plane. $\mathcal{E}_{z}$ produces a potential difference $\Delta_{z}=l \mathcal{E}_{z}$ between sub-lattices with a separation $l$. $\Delta_{\text {so }}$ is the band gap generated by intrinsic spin-orbit interaction and results a mass to the Dirac fermions $[15,16,17,12] . s= \pm 1$ stands for spin up and down, respectively. In the Landau 
gauge $\mathbf{A}=(-B y, 0,0)$, and the momentum operator can be written as $\hat{\mathbf{p}}_{i} \rightarrow \hat{\mathbf{p}}_{i}+q \mathbf{A}_{i}$. Therefore, the low energy effective Hamiltonian reads as

$$
\hat{H}_{s \xi}=\left(\begin{array}{cc}
\Delta_{s \xi} & v_{F}\left[\xi\left(\hat{p}_{x}-\frac{e}{c} B y\right)-i \hat{p}_{y}\right] \\
v_{F}\left[\xi\left(\hat{p}_{x}-\frac{e}{c} B y\right)+i \hat{p}_{y}\right] & -\Delta_{s \xi}
\end{array}\right),
$$

where

$$
\Delta_{s \xi}=\frac{1}{2}\left(\Delta_{z}-s \xi \Delta_{\mathrm{so}}\right)
$$

There exists a topological phase transition from the phase TI with $\left|\Delta_{z}\right|<\Delta_{\text {so }}$ to the phase BI with $\left|\Delta_{z}\right|>\Delta_{\text {so }}$. The effective gap $\left|\Delta_{s \xi}\right|$ becomes zero at the charge neutrality points $[13,29,30,31,32,20]$. The eigenvalue equation can be rewritten as $[15,16,17,12]$

$$
\hat{H} \psi=\epsilon \psi \text {. }
$$

The sublattices $A$ and $B$ are characterized by the components $\psi_{A}$ and $\psi_{B}$ of the wave function

$$
\psi=\left(\begin{array}{c}
\psi_{A} \\
\psi_{B}
\end{array}\right)
$$

The choice of the vector potential $\mathbf{A}=(-B y, 0,0)$ preserves the translational invariance along the $x$-axis. The eigenvalues and eigenfunctions take the form

$$
\epsilon_{n}^{s \xi}=\left\{\begin{array}{l}
\operatorname{sgn}(n) \sqrt{|n| \hbar^{2} \omega^{2}+\Delta_{s \xi}^{2}}, \quad n \neq 0, \\
-\xi \Delta_{s \xi}, \quad n=0
\end{array}\right.
$$

and

$$
|n\rangle_{s \xi}=\left(\begin{array}{c}
-i A_{n}^{s \xi}|| n\left|-\xi_{+}\right\rangle \\
B_{n}^{s \xi}|| n\left|-\xi_{-}\right\rangle
\end{array}\right)
$$

respectively. $\omega=v_{F} \sqrt{2 e B / \hbar}$ is the cyclotron frequency. $|n\rangle$ stands for the orthonormal Fock eigenstates of the harmonical oscillator with $n=0, \pm 1, \pm 2, \ldots$ and $\xi_{ \pm}=(1 \pm \xi) / 2$. The constants $A_{n}^{s \xi}$ and $B_{n}^{s \xi}$ in Eq. (7) are [20]

$$
\begin{aligned}
& A_{n}^{s \xi}= \begin{cases}\operatorname{sgn}(n) \sqrt{\frac{\left|E_{n}^{s \xi}\right|+\operatorname{sgn}(n) \Delta_{s \xi}}{2\left|E_{n}^{s \xi}\right|}}, \quad n \neq 0, \\
\xi_{-}, \quad n=0,\end{cases} \\
& B_{n}^{s \xi}= \begin{cases}\sqrt{\frac{\left|E_{n}^{s \xi}\right|-\operatorname{sgn}(n) \Delta_{s \xi}}{2\left|E_{n}^{s \xi}\right|}}, & n \neq 0, \\
\xi_{+}, & n=0\end{cases}
\end{aligned}
$$

The Fock states in position space have the form

$$
\langle x \mid n\rangle=\frac{\omega^{1 / 4}}{\sqrt{2^{n} n ! \sqrt{\pi}}} e^{-\omega x^{2} / 2} H_{n}(\sqrt{\omega} x),
$$

where $H_{n}$ are the Hermite polynomials of degree $n$. The density corresponding to the eigenstate $(7)$ reads as

$$
\rho_{n}^{s \xi}(x)=\left(A_{n}^{s \xi}\right)^{2}\left|\left\langle x|| n \mid-\xi_{+}\right\rangle\right|^{2}+\left(B_{n}^{s \xi}\right)^{2}\left|\left\langle x|| n \mid-\xi_{-}\right\rangle\right|^{2} .
$$


The momentum space Fock states are

$$
\gamma_{n}^{s \xi}(p)=\left(A_{n}^{s \xi}\right)^{2}\left|\left\langle p|| n \mid-\xi_{+}\right\rangle\right|^{2}+\left(B_{n}^{s \xi}\right)^{2}\left|\left\langle p|| n \mid-\xi_{-}\right\rangle\right|^{2}
$$

where

$$
\langle p \mid n\rangle=\frac{\omega^{-1 / 4}}{\sqrt{2^{n} n ! \sqrt{\pi}}} e^{-\omega x^{2} / 2} H_{n}(p / \sqrt{\omega}) .
$$

Note the similar forms of the position and momentum space densities. They differ only in the appearance of $\sqrt{\omega}$ : in the nominator (denominator) in the argument of the Hermite polynomials in position (momentum) space.

\section{Rényi-Fisher entropy product}

The Rényi entropy of order $\alpha$ for a probability density function $f(x)$ normalized to one is defined by [36]

$$
R_{f}^{\alpha} \equiv \frac{1}{1-\alpha} \ln \int f^{\alpha}(x) d x \quad \text { for } \quad 0<\alpha<\infty \quad \alpha \neq 1 .
$$

The limit $\alpha \rightarrow 1$ gives the Shannon entropy [37]

$$
S_{f} \equiv-\int f(x) \ln f(x) d x
$$

The Rényi entropy power of order $\alpha$ is defined by

$$
\begin{aligned}
& N_{f}^{\alpha} \equiv\left(\frac{\alpha}{2 \alpha-1}\right)^{\frac{2 \alpha-1}{\alpha-1}} \frac{1}{2 \pi} \exp \left(2 R_{f}^{\alpha}\right) \\
& =\beta^{1 /(1-\beta)} \frac{1}{2 \pi} \exp \left(2 R_{f}^{\alpha}\right),
\end{aligned}
$$

where $\beta$ is given by the equation $\alpha^{-1}+\beta^{-1}=2$. The limit $\alpha \rightarrow 1$ leads to the Shannon entropy power

$$
N_{f} \equiv \frac{1}{2 \pi e} e^{2 S_{f}}
$$

The Rényi entropy power has the property [39]

$$
N_{f}^{\alpha}>N_{f}^{\alpha^{\prime}} \quad \alpha<\alpha^{\prime} .
$$

The Rényi entropy and the Rényi entropy power can be constructed in both the position and the momentum spaces: $R_{\rho}^{\alpha}, N_{\rho}^{\alpha}, R_{\gamma}^{\alpha}$ and $N_{\gamma}^{\alpha}$, respectively, with the position $(\rho)$ and momentum $(\gamma)$ densities. The Rényi uncertainty relation [38] can be expressed in terms of Rényi entropy power in a compact form by

$$
N_{\rho}^{\alpha} N_{\gamma}^{\beta} \geq 1 / 4
$$

The Fisher information of the probability density function $f$ is given by $[40,41]$

$$
I_{f} \equiv \int \frac{1}{f(x)}\left(\frac{d f(x)}{d x}\right)^{2} d x
$$


The Stam uncertainty principle has the form [42]

$$
I_{\rho_{n}^{s \xi}} \leq 4 \sigma_{\gamma_{n}^{s \xi}}
$$

in position space and

$$
I_{\gamma_{n}^{s \xi}} \leq 4 \sigma_{\rho_{n}^{s \xi}}
$$

in momentum space. $\sigma_{f}$ stands for the variance of the density $f$.

The Rényi-Fisher information product is defined as

$$
P_{f}^{\alpha}=N_{f}^{\alpha} I_{f} \quad \text { with } \quad \alpha \in(1 / 2,1] .
$$

There exist an important inequality, the Rényi-Fisher relation

$$
P_{f}^{\alpha} \geq 1 \quad \text { with } \quad \alpha \in(1 / 2,1] \text {. }
$$

Combining inequalities (20), (21) and (23) we arive at the uncertainty relations [1]

$$
N_{\rho^{\alpha}} \geq \frac{1}{4 \sigma_{\gamma}}, \quad \alpha \in(1 / 2,1]
$$

and

$$
N_{\gamma}^{\alpha} \geq \frac{1}{4 \sigma_{\rho}}, \quad \alpha \in(1 / 2,1] .
$$

We calculated the Rényi entropy with the probability density functions $\rho_{n}^{s \xi}(10)$ and $\gamma_{n}^{s \xi}(11)$, respectively. We can take advantage of the fact that the densities in position and momentum spaces take almost the same form. The difference appears in the presence of $\sqrt{\omega}$ in the nominator (denominator) in the argument of the Hermite polynomials in position (momentum) space. Introducing the new variable $y=x \sqrt{\omega}$, it can be easily shown that

$$
R_{\rho_{n}^{s \xi}}^{\alpha}=\frac{1}{1-\alpha} \ln \left[\int_{-\infty}^{\infty}\left(\rho_{n}^{s \xi}(x)\right)^{\alpha} d x\right]=\tilde{R}_{\rho_{n}^{s \xi}}^{\alpha}-\frac{1}{2} \ln (\omega)
$$

and

$$
\tilde{R}_{\rho_{n}^{s \xi}}^{\alpha}=\frac{1}{1-\alpha} \ln \left[\int_{-\infty}^{\infty}\left(\tilde{\rho}_{n}^{s \xi}(y)\right)^{\alpha} d y\right]
$$

where $\tilde{\rho}_{n}^{s \xi}$ is the normalized density obtained after the variable change $y=x \sqrt{\omega}$.

The Rényi entropy power reads as

$$
N_{\rho_{n}^{s \xi}}^{\alpha}=\frac{1}{2 \pi \omega}\left(\frac{\alpha}{2 \alpha-1}\right)^{\frac{2 \alpha-1}{\alpha-1}}\left[\int_{-\infty}^{\infty}\left(\tilde{\rho}_{n}^{s \xi}(y)\right)^{\alpha} d y\right]^{2 /(1-\alpha)} .
$$

On the other hand, in the momentum space the Rényi entropy takes the form

$$
R_{\gamma_{n}^{s \xi}}^{\alpha}=\frac{1}{1-\alpha} \ln \left[\int_{-\infty}^{\infty}\left(\gamma_{n}^{s \xi}(p)\right)^{\alpha} d p\right]=\tilde{R}_{\gamma_{n}^{s \xi}}^{\alpha}+\frac{1}{2} \ln (\omega)
$$

where

$$
\tilde{R}_{\gamma_{n}^{s \xi}}^{\alpha}=\frac{1}{1-\alpha} \ln \left[\int_{-\infty}^{\infty}\left(\tilde{\gamma}_{n}^{s \xi}(y)\right)^{\alpha} d y\right]
$$

and $\tilde{\gamma}_{n}^{s \xi}$ is the normalized momentum density obtained after the variable change $y=x / \sqrt{\omega}$. 
The Rényi entropy power can be written as

$$
N_{\gamma_{n}^{s \xi}}^{\alpha}=\frac{\omega}{2 \pi}\left(\frac{\alpha}{2 \alpha-1}\right)^{\frac{2 \alpha-1}{\alpha-1}}\left[\int_{-\infty}^{\infty}\left(\tilde{\gamma}_{n}^{s \xi}(y)\right)^{\alpha} d y\right]^{2 /(1-\alpha)} .
$$

The Fisher information reads as

$$
I_{\rho_{n}^{s \xi}}=\int_{-\infty}^{\infty} \frac{\left(\left(\rho_{n}^{s \xi}(x)\right)^{\prime}\right)^{2}}{\rho_{n}^{s \xi}(x)} d x=\omega \int_{-\infty}^{\infty} \frac{\left(\left(\tilde{\rho}_{n}^{s \xi}(y)\right)^{\prime}\right)^{2}}{\tilde{\rho}_{n}^{s \xi}(y)} d y=\omega \tilde{I}_{\rho_{n}^{s \xi}}
$$

in position space and

$$
I_{\gamma_{n}^{s \xi}}=\omega^{-1} \int_{-\infty}^{\infty} \frac{\left(\left(\tilde{\gamma}_{n}^{s \xi}(y)\right)^{\prime}\right)^{2}}{\tilde{\gamma}_{n}^{s \xi}(y)} d y=\omega^{-1} \tilde{I}_{\gamma_{n}^{s \xi}}
$$

in momentum space.

The Rényi-Fisher product takes the form

$$
P_{\rho_{n}^{s \xi}}^{\alpha}=N_{\rho_{n}^{s \xi}}^{\alpha} I_{\rho_{n}^{s \xi}}=\left(\frac{\alpha}{2 \alpha-1}\right)^{\frac{2 \alpha-1}{\alpha-1}}\left[\int_{-\infty}^{\infty}\left(\tilde{\rho}_{n}^{s \xi}(y)\right)^{\alpha} d y\right]^{2 /(1-\alpha)} \tilde{I}_{\rho_{n}^{s \xi}}
$$

and

$$
P_{\gamma_{n}^{s \xi}}^{\alpha}=N_{\gamma_{n}^{s \xi}}^{\alpha} I_{\gamma_{n}^{s \xi}}=\left(\frac{\alpha}{2 \alpha-1}\right)^{\frac{2 \alpha-1}{\alpha-1}}\left[\int_{-\infty}^{\infty}\left(\tilde{\gamma}_{n}^{s \xi}(y)\right)^{\alpha} d y\right]^{2 /(1-\alpha)} \tilde{I}_{\gamma_{n}^{s \xi}}
$$

in position and momentum spaces, respectively. It follows form Eqs. (10) and (11) that $\tilde{\rho}_{n}^{s \xi}=\tilde{\gamma}_{n}^{s \xi}$. Therefore, $\tilde{I}_{\rho_{n}^{s \xi}}=\tilde{I}_{\gamma_{n}^{s \xi}}, \omega N_{\rho_{n}^{s \xi}}^{\alpha}=\omega^{-1} N_{\gamma_{n}^{s \xi}}^{\alpha}$ and $P_{\rho_{n}^{s \xi}}^{\alpha}=P_{\gamma_{n}^{s \xi}}^{\alpha}$. That is, the position and momentum space Rényi-Fisher products are equal. We determined the Rényi-Fisher products for several values of $\alpha$. Fig. 1. presents $P_{\rho_{n}^{s \xi}}^{\alpha}$ for the Landau levels $|n|=1,2,3,4$ as a function of $\Delta_{z} / \Delta_{s o}$ with $\alpha=0.75, s= \pm 1$ and $\xi=1$. The calculations presented here were done with $B=0.1 T$. Other values of $B$ resulted similar behaviour.

Then we calculated the sum of the Rényi-Fisher product for electrons and holes

$$
\hat{P}_{f_{n}^{s \xi}}^{\alpha}=P_{f_{n}^{s \xi}}^{\alpha}+P_{f_{-n}^{s \xi}}^{\alpha}
$$

for several values of $\alpha$. The combined Rényi-Fisher product $\hat{P}_{f_{n}^{s \xi}}^{\alpha}$ is plotted in Fig. 2 . for silicene as a function of the ratio $\Delta_{z} / \Delta_{\text {so }}$ for Landau levels $|n|=1,2,3,4$ for spin up and spin down in the valley $\xi=1$. In Eq. (36) $f$ stands either the position $(\rho)$ or the momentum $(\gamma)$ space density. The curves exhibit minimum at the charge neutrality points $\Delta_{z}=\Delta_{s o}\left(\Delta_{z}=-\Delta_{s o}\right)$ for spin up (down) case. Furthermore, we can also observe that the slopes of the spin up and down curves have the same sign in the phase BI $\left(\left|\Delta_{z}\right|>\Delta_{\text {so }}\right)$ and have different sign in the phase TI $\left(\left|\Delta_{z}\right|<\Delta_{\text {so }}\right)$. Analogously to the Chern number, based on the combined Rényi-Fisher product we can define a topological quantum number to identify the different phases:

$$
C_{f_{n}^{s \xi}}^{1}=\operatorname{sgn}\left(\frac{\partial \hat{P}_{f_{n}^{s \xi}}^{\alpha}}{\partial \Delta_{z}} \frac{\partial \hat{P}_{f_{n}^{-s \xi}}^{\alpha}}{\partial \Delta_{z}}\right) .
$$

This quantity can be considered a topological charge:

$$
C_{f_{n}^{s \xi}}^{1}\left(\Delta_{z}\right)=\left\{1,\left|\Delta_{z}\right|>\Delta_{S O} \quad B I-1,\left|\Delta_{z}\right|<\Delta_{S O} \quad T I .\right.
$$



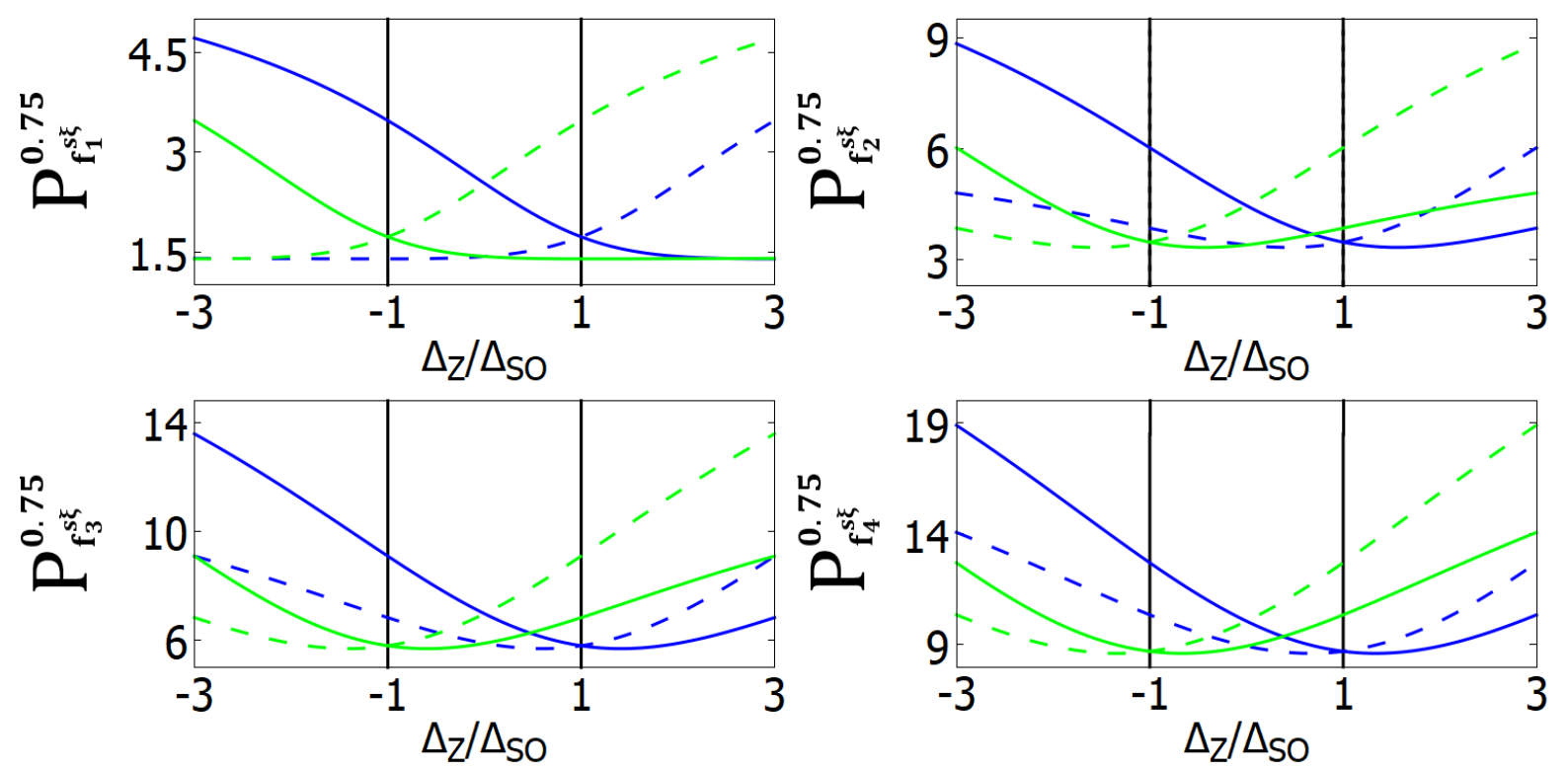

Figure 1. Rényi-Fisher product for the Landau levels $|n|=1,2,3,4$, as a function of $\Delta_{z} / \Delta_{\text {so }}$ for spin up (blue lines) and spin down (green lines) and $\alpha=0.75$ in the valley $\xi=1$. Solid (dashed) lines represent electrons (holes). $f_{n}$ stands for the Landau levels $|n|$.
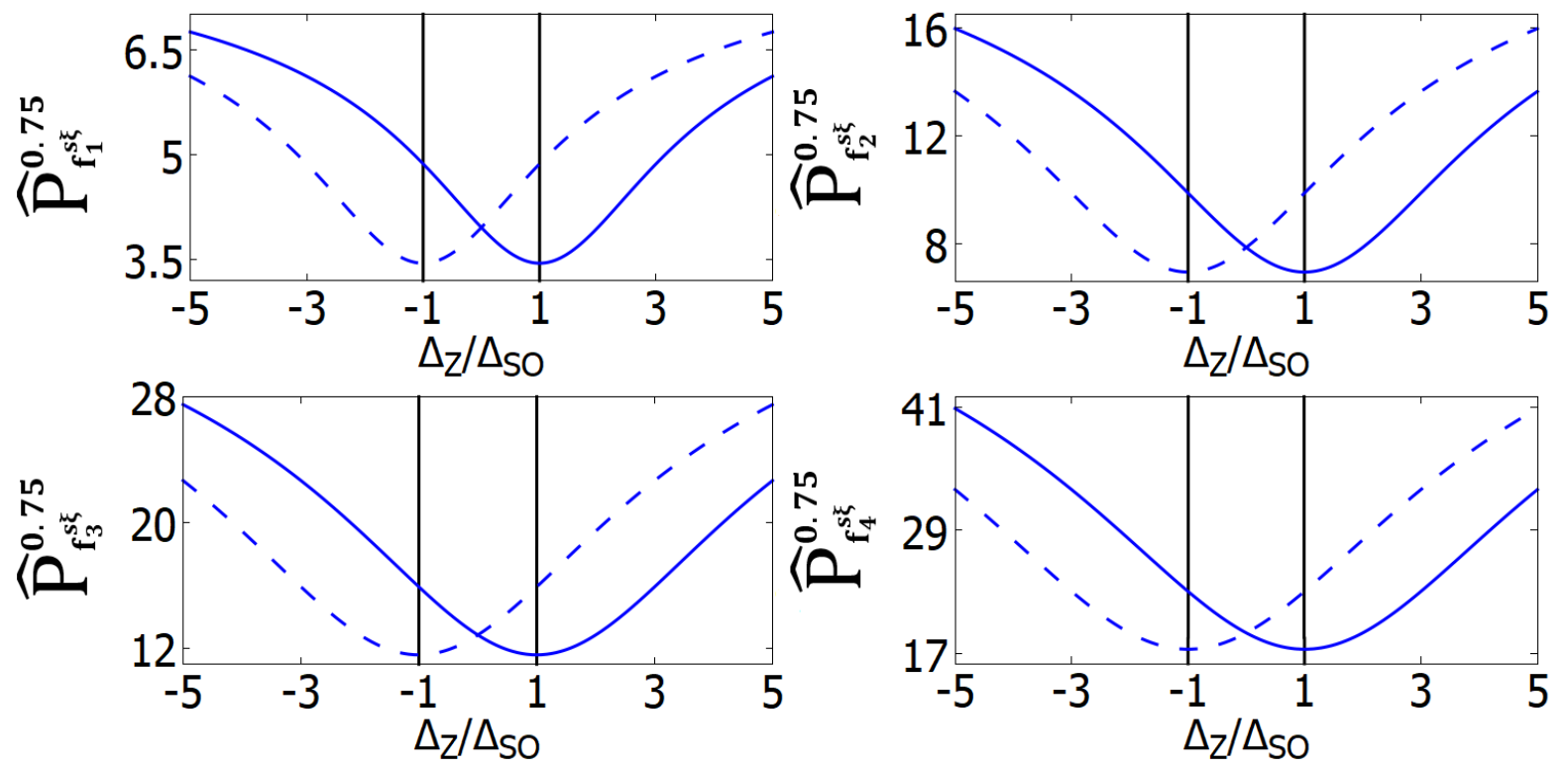

Figure 2. Combined Rényi-Fisher product for the Landau levels $|n|=1,2,3,4$, as a function of $\Delta_{z} / \Delta_{s o}$ for spin up (solid lines) and spin down (dashed lines) and $\alpha=0.75$ in the valley $\xi=1$. $f_{n}$ stands for the Landau levels $|n|$. 


\section{Stam-Rényi uncertainty relations}

Consider now the uncertainty relation (24) and define the differences

$$
S_{\rho_{n}^{s \xi}}^{\alpha}=N_{\rho_{n}^{s \xi}}^{\alpha}-\frac{1}{4 \sigma_{\gamma_{n}^{s \xi}}} \leq 0
$$

and

$$
S_{\gamma_{n}^{s \xi}}^{\alpha}=N_{\gamma_{n}^{s \xi}}^{\alpha}-\frac{1}{4 \sigma_{\rho_{n}^{s \xi}}} \leq 0 .
$$

in position and momentum spaces, respectively. Then the sum of these differences are constructed for electrons and holes:

$$
\hat{S}_{\rho_{n}^{s \xi}}^{\alpha}=S_{\rho_{n}^{s \xi}}^{\alpha}+S_{\rho_{-n}^{s \xi}}^{\alpha} \leq 0
$$

and

$$
\hat{S}_{\gamma_{n}^{s \xi}}^{\alpha}=S_{\gamma_{n}^{s \xi}}^{\alpha}+S_{\gamma_{-n}^{s \xi}}^{\alpha} \leq 0 .
$$

Eqs. (28) and (29) lead to

$$
\omega \hat{S}_{\rho_{n}^{s \xi}}^{\alpha}=\omega^{-1} \hat{S}_{\gamma_{n}^{s \xi}}^{\alpha} .
$$

$\omega \hat{S}_{\rho_{n}^{s \xi}}^{\alpha}$ is plotted in Fig. 3. for silicene as a function of the ratio $\Delta_{z} / \Delta_{s o}$ for Landau levels $|n|=1,2,3,4$ for spin up and spin down in the valley $\xi=1$. We obtained exactly the same figure for $\omega^{-1} \hat{S}_{\gamma_{n} \xi}^{\alpha}$. The curves exhibit maxima at the charge neutrality points $\Delta_{z}=\Delta_{s o}\left(\Delta_{z}=-\Delta_{s o}\right)$ for spin up (down) case. Furthermore, the slopes of the spin up and down curves possess the same sign in the phase BI $\left(\left|\Delta_{z}\right|>\Delta_{\text {so }}\right)$ and different sign in the phase TI $\left(\left|\Delta_{z}\right|<\Delta_{\text {so }}\right)$. Consequently, we can construct another topological quantum number that detects the different phases as the Chern number does:

$$
C_{\rho_{n}^{s \xi}}^{2}=\operatorname{sgn}\left(\frac{\partial \hat{S}_{\rho_{n}^{s \xi}}^{\alpha}}{\partial \Delta_{z}} \frac{\partial \hat{S}_{\rho_{n}^{-s \xi}}^{\alpha}}{\partial \Delta_{z}}\right) .
$$

This quantity can also be recognized as a topological charge:

$$
C_{\rho_{n}^{s \xi}}^{2}\left(\Delta_{z}\right)=\left\{1,\left|\Delta_{z}\right|>\Delta_{S O} \quad B I-1,\left|\Delta_{z}\right|<\Delta_{S O} \quad T I .\right.
$$

Moreover, we can immediately see that this topological quantum number can be obtained in momentum space, as well:

$$
C_{\gamma_{n}^{s \xi}}^{2}=\operatorname{sgn}\left(\frac{\partial \hat{S}_{\gamma_{n}^{s \xi}}^{\alpha}}{\partial \Delta_{z}} \frac{\partial \hat{S}_{\gamma_{n}^{-s \xi}}^{\alpha}}{\partial \Delta_{z}}\right)=C_{\rho_{n}^{s \xi}}^{2} .
$$

Define now the Stam-Rényi uncertainty products as

$$
T_{\rho_{n}^{s \xi}}^{\alpha}=N_{\rho_{n}^{s \xi}}^{\alpha} 4 \sigma_{\gamma_{n}^{s \xi}}
$$

and

$$
T_{\gamma_{n}^{s \xi}}^{\alpha}=N_{\gamma_{n}^{s \xi}}^{\alpha} 4 \sigma_{\rho_{n}^{s \xi}}
$$



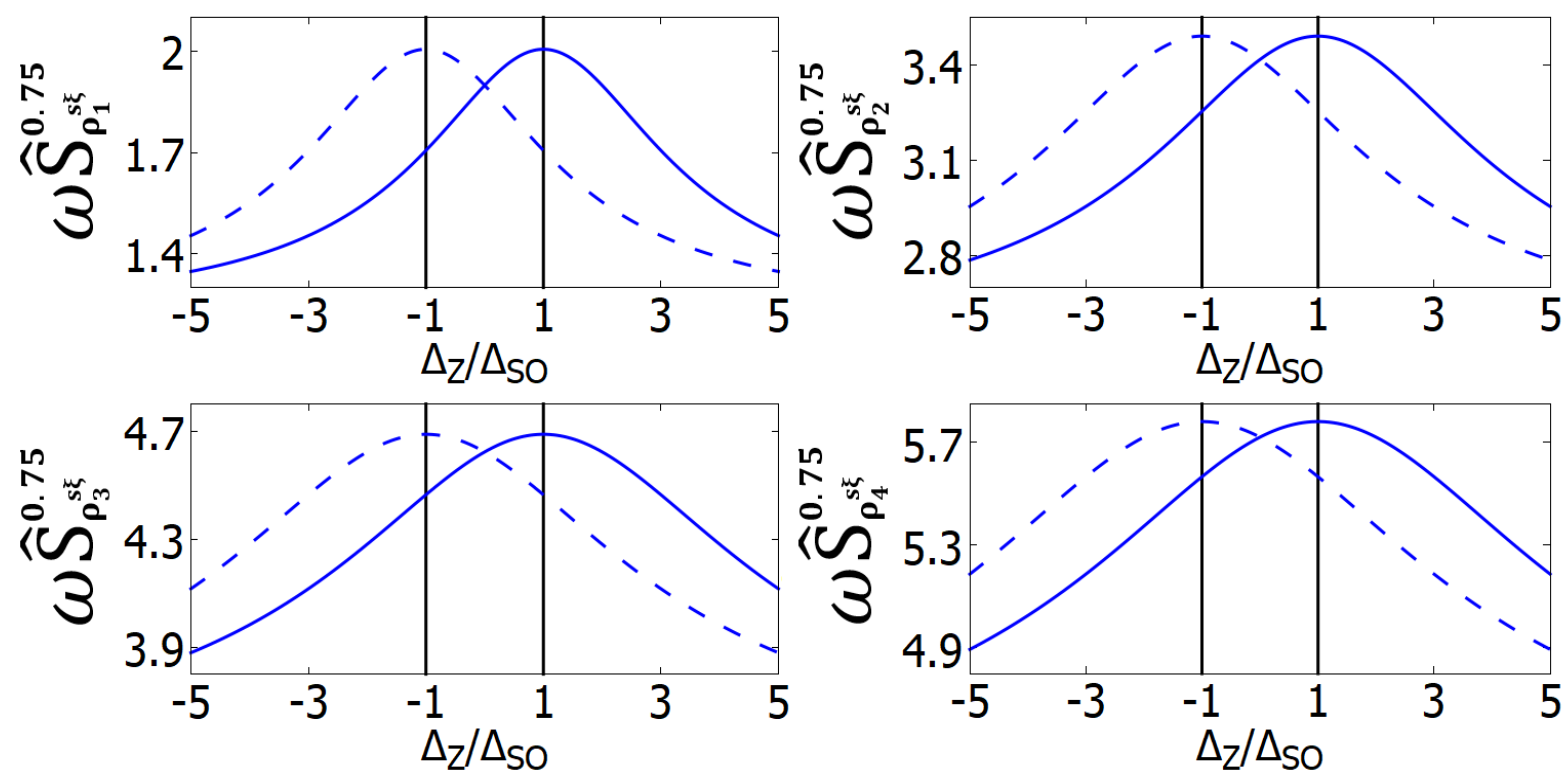

Figure 3. Combined difference $\hat{S}_{\rho_{n}^{s \xi}}^{\alpha}$ for electrons and holes as a function of $\Delta_{z} / \Delta_{s o}$ for Landau levels $|n|=1,2,3,4$ for spin up (solid lines) and spin down (dashed lines) in the valley $\xi=1$ in case of $\alpha=0.75$.

In the previous section we proved that $P_{\rho_{n}^{s \xi}}^{\alpha}=P_{\gamma_{n}^{s \xi}}^{\alpha}$. A similar proof can be given to the the assertion $T_{\rho_{n}^{s \xi}}^{\alpha}=T_{\gamma_{n}^{s \xi}}^{\alpha}$. Combining the relations (22), (23), (24) and (25) we arrive at the inequalities:

$$
1 \leq P_{f_{n}^{s \xi}}^{\alpha} \leq T_{f_{n}^{s \xi}}^{\alpha}
$$

That is, the Stam-Rényi uncertainty products $T_{f_{n}^{s \xi}}^{\alpha}$ act as an upper limit to the RényiFisher entropy products $P_{f_{n}^{s \xi}}^{\alpha}$. Now, we construct the combined Stam-Rényi uncertainty product for holes and electrons:

$$
\hat{T}_{f_{n}^{s \xi}}^{\alpha}=T_{f_{n}^{s \xi}}^{\alpha}+T_{f_{-n}^{s \xi}}^{\alpha}
$$

In Eq. (50) $f$ stands either the position $(\rho)$ or the momentum $(\gamma)$ space density. The combined Stam-Rényi uncertainty product $\hat{T}_{f_{n}^{s \xi}}^{\alpha}$ is plotted in Fig. 4. for silicene as a function of the ratio $\Delta_{z} / \Delta_{s o}$ for Landau levels $|n|=1,2,3,4$ for spin up and spin down in the valley $\xi=1$. The curves exhibit maxima at the charge neutrality points $\Delta_{z}=\Delta_{\text {so }}$ $\left(\Delta_{z}=-\Delta_{s o}\right)$ for spin up (down) case. Furthermore, we can also observe that the slopes of the spin up and down curves have the same sign in the phase BI $\left(\left|\Delta_{z}\right|>\Delta_{\text {so }}\right)$ and have different sign in the phase TI $\left(\left|\Delta_{z}\right|<\Delta_{\text {so }}\right)$. Consequently, we can define a topological quantum number (similar to the Chern number) to identify the different phases utilizing the combined Stam-Rényi uncertainty products:

$$
C_{f_{n}^{s \xi}}^{3}=\operatorname{sgn}\left(\frac{\partial \hat{T}_{f_{n}^{s \xi}}^{\alpha}}{\partial \Delta_{z}} \frac{\partial \hat{T}_{f_{n}^{-s \xi}}^{\alpha}}{\partial \Delta_{z}}\right) .
$$



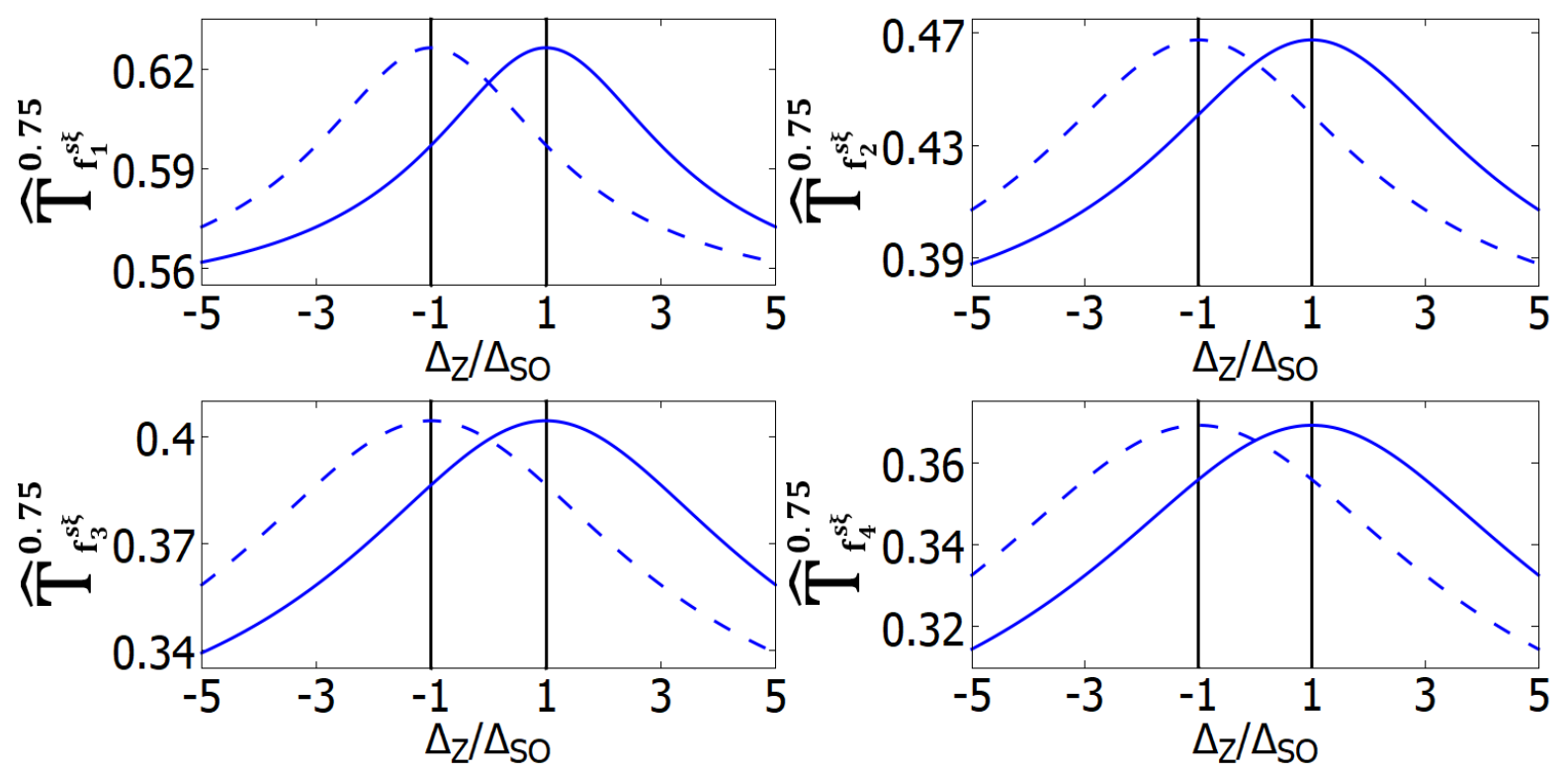

Figure 4. Combined Stam-Rényi uncertainty products $\hat{T}_{f_{n}^{s \xi}}^{\alpha}$ for electrons and holes as a function of $\Delta_{z} / \Delta_{\text {so }}$ for Landau levels $|n|=1,2,3,4$ for spin up (solid lines) and spin down (dashed lines) in the valley $\xi=1$ in case of $\alpha=0.75$.

This quantity can be recogized as a topological charge:

$$
C_{f_{n}^{s \xi}}^{3}\left(\Delta_{z}\right)=\left\{1,\left|\Delta_{z}\right|>\Delta_{S O} \quad B I-1,\left|\Delta_{z}\right|<\Delta_{S O} \quad T I\right.
$$

\section{Conclusions}

Rényi-Fisher entropy product has here been presented as a marker of topological phase transitions in silicene. In this 2D Dirac gapped material, there is a topological phase transition from the phase TI with $\left|\Delta_{z}\right|<\Delta_{\text {so }}$ to the phase BI with $\left|\Delta_{z}\right|>\Delta_{\text {so }}$. The Rényi-Fisher entropy product of electrons plus holes displays minimum at the charge neutrality points $\Delta_{z}=\Delta_{s o}\left(\Delta_{z}=-\Delta_{s o}\right)$ for spin up (down) case. This quantity can be used to define a topological quantum number capable to identify the different phases. We have proved that the position and momentum space Rényi-Fisher entropy products are equal. We have constructed the Stam-Rényi difference of the electrons plus holes that shows a maximum at the charge neutrality points. This quantity is also suitable for composing a topological quantum number. We have also defined the StamRényi uncertainty poduct that has the same value in both the position and mometum spaces. Furthermore, it provides an upper bound to the Rényi-Fisher entropy product. Relation (49) gives upper and lower bounds for the Rényi-Fisher entropy product. We have shown that the combined Stam-Rényi uncertainty poduct for electrons plus holes exhibit a maximum at the charge neutrality points. This quantity has been utilized in defining a topological quantum number. 


\section{Acknowledgments}

The work was supported by the Junta de Andalucía projects P12-FQM.1861 and FQM381 and the National Research, Development and Innovation Fund of Hungary, financed under the 123988 funding scheme and the EU-funded Hungarian grant EFOP-3.6.2-162017-00005.

[1] E. Romera and Á. Nagy, Phys. Lett. A 372, 6823 (2008).

[2] C. L. Kane, and E. J. Mele, Phys. Rev. Lett. 95, 226801 (2005).

[3] B. Andrei Bernevig, Taylor L. Hughes, and Shou-Cheng Zhang, Science 314, 1757-1761 (2006).

[4] X-L. Qui and S-C. Zhang, Physics Today 63, 33 (2010).

[5] K. Takeda, K. Shiraishi, Phys. Rev. B 50 (1994) 14916.

[6] G. G. Guzman-Verri, L. Lew Yan, Phys. Rev. B 76 (2007) 075131.

[7] P. Vogt et al., Phys. Rev. Lett. 108 (2012) 155501.

[8] B. Augray, A. Kara, S. B. Vizzini, H. Oughaldou, C. LéAndri, B. Ealet, G. Le Lay, App. Phys. Lett. 96 (2010) 183102.

[9] B. Lalmi, H. Oughaddou, H. Enriquez, A. Kara, S. B. Vizzini, B. N. Ealet, B. Augray, App. Phys. Letters 97 (2010) 223109.

[10] A. Feurence, R. Friedlein, T. Ozaki, H. Kawai, Y. Wang, Y. Y. Takamura, Phys. Rev. Lett. 108 (2012) 245501.

[11] P. E. Padova et al., App. Phys. Lett. 96 (2010) 261905.

[12] W-F. Tsai, C-Y. Huang, T-R Chang et al. Nat. Commun. 4, 1500 (2013)

[13] M. Tahir, U. Schwingenschlögl, Scientific Reports, 3, 1075 (2013).

[14] B. Andrei Bernevig, Taylor L. Hughes and Shou-Cheng Zhang, Science 314, 1757-1761 (2006).

[15] N. D. Drummond, V. Zólyomi, and V. I. Fal'ko, Phys. Rev. B 85, 075423 (2012).

[16] C. C. Liu, W. Feng, and Y. Yao, Phys. Rev. Lett. 107, 076802 (2011).

[17] C. C. Liu, H. Jiang, and Y. Yao, Phys. Rev. B 84, 195430 (2011).

[18] S. Trivedi, A. Srivastava and R. Kurchania, J. Comput. Theor. Nanosci. 11, 1-8 (2014). doi:10.1166/jctn.2014.3428

[19] B van den Broek et al. 2D Materials 1 (2014) 021004. doi:10.1088/2053-1583/1/2/021004

[20] L. Stille, C. J. Tabert, and E. J. Nicol, Phys. Rev. B 86, 195405 (2012); C.J. Tabert and E.J. Nicol, Phys. Rev. Lett. 110, 197402 (2013); C.J. Tabert and E.J. Nicol, Phys. Rev. B 88, 085434 (2013).

[21] M. Ezawa, New Journal of Physics 14 (2012) 033003

[22] S. J. Gu, Int. J. of Mod. Phys. B 24, 4371 (2010).

[23] Wu L. A.; Sarandy M. S.; Lidar D. A.; Sham L. J. Phys. Rev. A 74, 052335 (2006).

[24] Nagy, Á.,; Calixto M.,; Romera, E., J. Chem. Theory Comput. 9, 1068 (2013).

[25] Á. Nagy, E. Romera, Phys. Rev. A 88, 042515 (2013).

[26] S. J. Gu, Chi. Phys. Lett. 26, 026401 (2009).

[27] E. Romera and Á. Nagy, Phys. Lett. A 377, 3098 (2013).

[28] Á. Nagy and E. Romera, Europhys. Lett. bf 109 (2015) 60002.

[29] E. Romera and M. Calixto, EPL, 111, 37006 (2015).

[30] M. Calixto and E. Romera, Journal of Statistical Mechanics, P06029 (2015).

[31] E. Romera and M. Calixto, Journal of Physics: Condensed Matter,27, 175003 (2015).

[32] M. Calixto and E. Romera, EPL, 109, 40003 (2015).

[33] J. C. Bolívar, Á. Nagy, and E. Romera, Fisher information and topological insulator phase transitions, Preprint (2017).

[34] J. C. Bolívar, E. Romera, Phys. Lett. A 381, 1753 (2017).

[35] E. Romera, J. C. Bolívar, J B. Roldán, F. de los Santos, Europhys. Lett. 115, 20008 (2016).

[36] A. Rényi, in Proceedings of Fourth Berkeley Symp. on Mathematics, Statistics and Probability, vol.1. Univ. California Press, Berkeley, 1961, pp.547. 
[37] C. E. Shannon, Bell Syst. Tech. J. 27, 379 (1948).

[38] I. Bialynicki-Birula, Phys. Rev. A 74, 052101 (2006 ).

[39] A. Dembo, T. M. Cover, and J. A. Thomas, IEEE Trans. Inf. Theory 37, 1501 (1991).

[40] R. A. Fisher, Proc. Cambridge Philos. Soc.22 700 (1925).

[41] B. R. Frieden, Physics from Fisher Information. A unification; Cambridge: U. P., 1998.

[42] A. J. Stam, Inf. Cont. 2, 101 (1959). 